\title{
The Application of Magnetic Resonance Spectroscopy to Investigate the Effect of a Commercial Energy Drink
}

\author{
Tracy L. Burrows ${ }^{1^{*}}$, Kirrilly M. Pursey ${ }^{1}$ and Peter Stanwell ${ }^{1}$ \\ ${ }^{1}$ School of Health Sciences, Faculty of Health and Medicine, University of Newcastle, Callaghan, \\ NSW, Australia. \\ Authors' contributions \\ This work was carried out in collaboration between all authors. Authors TLB and PS designed the \\ study. Author KMP collected data. All authors read and approved the final manuscript.
}

Article Information

DOI: 10.9734/EJNFS/2015/9229

Original Research Article

Received $28^{\text {th }}$ January 2014

Accepted $14^{\text {th }}$ September 2014

Published $17^{\text {th }}$ December 2014

\section{ABSTRACT}

Aims: Proton magnetic resonance spectroscopy (MRS) is an imaging technique that provides noninvasive, in-vivo measurement of brain biochemistry. The objective of this study was to explore the effects of a commercial energy drink (ED) on brain metabolites in adult males using MRS.

Methods: Ten males (18 to 30 years), of mean body mass index $23.8 \pm 2.2 \mathrm{~kg} / \mathrm{m}^{2}$ [range $21.4-27.8$ $\left.\mathrm{kg} / \mathrm{m}^{2}\right]$ were recruited and randomized to either the intervention group $(n=5)$ or control group $(n=5)$. The intervention group were asked to consume two $250 \mathrm{ml}$ cans of Red Bull and the control group consumed no drink. One dimensional MRS on a 1.5T Achieva MRI scanner was obtained of bioccipital gray matter. Subjects were placed in the scanner and a spectrum acquired every 5.75 minutes for 60 minutes. Linear mixed models were generated to determine the main effects due to differences between groups and times and the interaction.

Results: There was a significant group by time effect for $\mathrm{N}$-acetylaspartate (NAA) $(P=.02, d=0.7)$ and a statistical trend for macromolecule 20 (MM20) $(P=.06 d=0.1)$ with the control group having higher concentrations than that of the intervention. Mean differences from baseline to 45 minutes for NAA in the intervention group were $-0.8 \mathrm{mM}(-12 \%)$ compared with $0.15 \mathrm{mM}(<1 \%)$ for control and for MM20 $-0.12 \mathrm{mM}(<1 \%)$ in the intervention group compared with $0.03 \mathrm{mM}(<1 \%)$ for control.

Conclusions: Consumption of two cans of ED was found to produce few changes in brain metabolites with moderate effect sizes. Changes detected in this study demonstrate the sensitivity 
of the MRS method to explore nutrition related variables with $1.5 \mathrm{~T}$ clinical MRI unit. The study provides some insight into an understudied, topical area of research.

Keywords: Energy drink; magnetic resonance spectroscopy; MRS.

\section{INTRODUCTION}

Proton magnetic resonance spectroscopy (MRS) is a technique to investigate brain metabolism invivo and to study endogenous biochemistry in the human brain non-invasively. It is capable of assessing the neurochemical profile of in-vivo brain tissue and thus provides biomarkers of neurological and metabolic disorders [1,2,3,4]. Disregarding the use in clinical studies, this methodology has not been widely used in the field of experimental nutrition, primarily due to its high cost, the need for specialized laboratory facilities and trained staff [5]. A small amount of previous diet related research using MRS has aimed to explore the pharmacologic effects of intravenous nutrient supplementation on the brain in-situ $[6,7]$ and has been used for rapid assessment of food ingredient monitoring and surveillance [8]. However, the effects of whole foods or beverages on human health which are consumed as part of an individual's regular dietary intake has not yet been explored using MRS. The non-invasive quality of this technique makes it suitable not only for diagnostic purposes but also exploratory studies. This includes examining the health effects of new age foods and beverages such as energy drinks (ED), where the effects of their short and long term consumption are not yet known and their health claims highly debated.

Energy drinks are new age beverages and their market size and consumption rates are growing at exponential rates, with males aged 18 to 34 years being the highest consumers [9]. These beverages are characterized by ingredients purported to enhance energy metabolism and to improve physical and cognitive performance [912]. An increasing number of publications are emerging from health authorities [13,14] and case reports [15-20] regarding the health implications associated with consumption of ED's often in combination with alcohol [21-25] and their unique combination of ingredients. The safety of ED's is not well substantiated in published literature and regulation is extremely variable worldwide [26].
A systematic review identified that few studies $(n=15)$ have investigated the implications associated with the consumption of the whole ED drink, with very few investigating effects of short and long term consumption [26]. In a second review it was reported that apart from one of the major ingredients, caffeine, there is weak evidence to substantiate nutritional health claims associated with ED [27]. Most studies have investigated individual ingredients, with caffeine the most commonly studied. Other common ED ingredients which vary widely across the spectrum of ED's include taurine, B-vitamins, glucose and herbal supplements including guarana and are often added in quantities that exceed recommended dietary intakes $[9,28]$. Interestingly, some of these ED ingredients such as taurine are present in human tissue and are able to be directly detected in the brain using invivo MRS. Due to homeostatic mechanisms, the basal levels of major brain metabolites are not expected to increase after ED consumption. However, a small pilot study [29] which investigated brain chemistry using MRS reported significant increases in taurine following ED consumption. These results are yet to be substantiated.

The objective of this exploratory study was to explore the effects of the commercial energy drink on the concentration of the metabolite taurine in the brain as well as other metabolites surviving specified statistical thresholds. A secondary aim was to demonstrate the application of novel imaging techniques such as MRS to the field of nutrition.

\section{METHODS}

Ten males aged 18 to 30 years were recruited from June to July, 2011. Exclusion criteria was consistent with MRS scanning procedures which included metal implants, pre-existing medical conditions and body mass $>150 \mathrm{~kg}$. Young adult male participants were recruited to limit potential confounding factors such as gender differences in brain metabolites which have been previously reported in brain MRS including males having less $\mathrm{N}$-acetylaspartate (NAA) when compared to females [30]. Eligible participants were invited to 
participate and voluntary, written informed consent was obtained. Participants were randomized to either the intervention group $(n=5)$ or control group $(n=5)$. The intervention group were asked to consume two $250 \mathrm{ml}$ cans of a commercially available ED, Red Bull $(160 \mathrm{mg}$ caffeine, $2000 \mathrm{mg}$ taurine, $1200 \mathrm{mg}$ glucuronolactone), and the control group consumed no ED drink. The study was approved by the University of Newcastle Human Research Ethics Committee. The dosage of Red Bull was informed by a pilot MRS study conducted by research staff (data not shown) and falls within the Australian safe consumption guidelines for caffeine [31] and therefore unlikely to cause any adverse events to participants. To ensure a consistent washout period, participants were asked to fast for two hours before their scanning session (apart from water), and refrain from caffeine intake (tea, coffee, cola drinks, chocolate and ED) for ten hours before scanning [32]. This is in line with previous studies and standard imaging protocols [33].

Prior to scanning, participants completed a 15item questionnaire regarding their usual consumption of ED adapted from a previously conducted study [34]. Basic demographic information (age, socio-economic status, ethnicity and occupation), frequency of consumption and types of EDs consumed were assessed. Socioeconomic status was calculated using the Socio Economic Indexes for Areas (SEIFA) deciles whereby postal codes receive a score of 1-10 with 1 being the lowest socioeconomic decile and 10 the highest [35]. ED consumption patterns with alcohol and the incidence of subjective adverse effects following the consumption of ED's were also assessed.

\subsection{MRS Protocol}

Participants attended a single scan session between the hours of 14:00 to 17:00 hours due to scanner availability and to reduce any possible influence associated with Circadian rhythms as previously described [36]. Depending on their group allocation, participants consumed either two cans of Red Bull (500 ml total) or consumed no drink, followed by MRI scan. Compliance of the consumption of the Red Bull was monitored. Participants remained in a rested state in the supine position for standardized ten minutes prior to scanning to ensure participants were assessed in the rested state.
One dimensional (1D) MRS on a 1.5T Achieva magnetic resonance imaging (MRI) scanner (Phillips, The Netherlands) using a phase-array head coil was obtained of bi-occipital gray matter. Subjects were placed in the scanner and a spectrum acquired every 5.75 minutes for 60 minutes. MR spectra were acquired with a pointresolved spectroscopy (PRESS), short echo time (TE) sequence (TR $1.5 \mathrm{~s}$, TE 35 ms, 192 signal averages) to maximize visibility of neurochemicals [37]. Ten spectra were acquired sequentially (i.e. temporal resolution of 5 minutes, 45 seconds) for a total examination time of approximately 60 minutes. This time point was chosen based on a previous published study [29] and our own preliminary data to confirm feasibility (data not shown).

A baseline spectrum was obtained for the intervention group (i.e. before consuming ED) followed by nine further sequential spectroscopy acquisitions. In the case of non-ED drinkers, 10 sequential spectroscopy acquisitions were acquired. Following each examination, raw spectroscopy data was exported offline and analysed with LCModel [38] using an appropriate metabolite basis set for the chosen examination parameters. LCModel analysis allows for the incorporation of metabolite a-priori knowledge to maximise quality of spectral fit. Prior knowledge methods in LCModel take into account the type of imager, magnetic field strength, echo time, and repetition time to maximise accuracy of quantification through incorporation of known signal parameters, such as relative frequencies, amplitude ratios, scalar coupling, and phases of resonances, which are characteristics of individual metabolites.

Spectroscopy processing incorporated water scaling using a non-water suppressed reference spectrum for signal quantification. LCModel initially reported on 70 single or combined metabolite resonances, however the majority of these were reported with Cramer-Rao lower bounds (CRLB) $>20 \%$ and were thus excluded from further analysis. The concentrations in this study are reported as absolute values in $\mathrm{mmol} / \mathrm{kg}$.

The CRLB values report on the variance of unbiased estimators and thus provides an assessment of noise-related errors on estimated parameters and, are thus indispensable in assessing reliability of the metabolite concentration estimates and providing a metric of quantification precision [8]. Quantification errors 
are caused by measurement noise and inadequate modelling of the overlapping background and other nuisance signals (residual water, lineshape distortions, ignoring metabolites with very small concentrations, etc). Quantitative results for signals attributable to glutamate (Glu), choline-containing compounds (tCho), Nacetylaspartate (NAA), Taurine, myo-inositol $(\mathrm{ml})$, and glutamate+glutamine $(\mathrm{Glx})$ were only reported if the corresponding Cramer-RaoLower-Bounds (CRLB values), calculated by LCModel, for spectral fit were $<15 \%$, while quantitative results for several macromolecules (MM09 and MM20) were only reported if the CRLB values were $\leq 20 \%$.In this study separate values for Glu, tCho, NAA, ml and Glx (<15\%) and MM09 and MM20 ( $\leq 20 \%)$ were chosen due the short T2 relaxation time (and hence broader signal) expected in these macromolecules.

\subsection{Statistical Analysis}

Metabolite concentrations were exported to SPSS (IBM, Version 19) and analyzed using descriptive statistics with $P<.05$ considered statistically significant. Linear mixed models were generated to determine the main effects due to differences between groups and times and also the group by time interaction. No imputation of missing values was carried out for subjects; the study and subjects were analyzed in their allocated, randomization group. The effects were time (0-60 minutes), group (intervention or control). Cohensd was used to compare effect sizes across different metabolites [39] and allows for a more direct comparison of effects on each outcome variable. These were calculated using the mean difference and the pooled standard deviation of the group $\left(d=M_{1}-M_{2} / \sigma_{\text {pooled }}\right)$.

\section{RESULTS}

Ten males were recruited and completed the study. The mean age of the participants was 23 years (range 22-25 years). Mean body mass index (BMI) of the sample was $23.8 \pm 2.2 \mathrm{~kg} / \mathrm{m}^{2}$ [21.4-27.8 $\left.\mathrm{kg} / \mathrm{m}^{2}\right]$ with the majority of participants $(n=7)$ classified as of healthy weight BMI category using World Health Organization BMI cut points [40]. All participants were Australian citizens and none identified as indigenous descent. The majority of participants $(n=7)$ were students and, the mean SEIFA decile was calculated to be 6.5 (range 2-9) and classified as moderate socio-economic status. No adverse reactions were recorded.

\subsection{ED Consumption}

Of the ten participants, six reported being regular consumers of ED's with an average consumption of one drink per week. The remaining four reported to be non-regular consumers of ED. There were no statistically significant differences in ED consumption patterns or anthropometric variables between the intervention and control groups at baseline. All consumers reported the use of ED's with alcohol and increased consumption during periods when in combination with alcohol. Of the non-ED consumers group, the primary reason prioritized for nonconsumption was the concern about the high caloric density of the beverage $(n=3)$ followed by taste $(n=1)$. Red Bull was the most commonly consumed ED of choice $(n=7)$, followed by $V$ Drink (Frucor Beverages, $n=2$ ), and Mother (Coca Cola Amatil, $n=1$ ). All consumers of ED reported the use of original varieties (full sugar) of ED's with only one participant reporting drinking sugar-free ED options. Reasons prioritized for the consumption of ED's included to improve physical energy and stamina $(n=3)$, prolong wakefulness $(n=2)$, and to improve mental acuity/concentration $(n=1)$. Insomnia and heart palpitations were the most frequently reported subjective adverse effects ( $n=3$ each).

\subsection{MRS Outcomes}

Brain metabolite concentrations at each time point are shown in Table 1, example spectra shown in Fig. 1. There were no significant differences between groups at baseline for measured brain metabolites. Taurine was not reliably detected in this study as evidenced by CRLB $>20 \%$.

The acquired set of metabolites in this study for both the intervention group and our own control data were within similar ranges to other published studies using a $1.5 \mathrm{~T}$ magnet $[41,42]$.

Linear mixed models showed there was a significant group by time effect for the metabolite NAA $\quad(P=.02, d=0.7)$ (Fig. 2a) and a statistical trend for MM20 ( $P=.06, d=0.1)$ (Fig. 2b) with the control group having higher concentrations than that of the intervention. Mean differences baseline to 60 minutes for NAA in the intervention group were $-0.8 \mathrm{mM} \quad(-12 \%)$ compared with $0.15 \mathrm{mM}(<1 \%)$ for control and for MM20 $-0.12 \mathrm{mM}(<1 \%)$ for the intervention group compared with $0.03 \mathrm{mM}(<1 \%)$ for control. 
Table 1. Brain metabolite concentrations $(\mathrm{mM} / \mathrm{kg})$ by group and time

\begin{tabular}{|c|c|c|c|c|c|c|c|c|c|c|c|c|c|c|c|c|}
\hline \multirow{2}{*}{$\begin{array}{l}\text { Time } \\
\text { (Mins) }\end{array}$} & \multicolumn{2}{|c|}{ Glu } & \multicolumn{2}{|c|}{ Ins } & \multicolumn{2}{|c|}{ NAA } & \multicolumn{2}{|c|}{ tCho } & \multicolumn{2}{|c|}{$\mathrm{tCr}$} & \multicolumn{2}{|c|}{ GluGIn (GIx) } & \multicolumn{2}{|c|}{ MM09 } & \multicolumn{2}{|c|}{ MM20 } \\
\hline & Control & Int & Control & Int & Control & Int & Control & Int & Control & Int & Control & Int & Control & Int & Control & Int \\
\hline 0 & $6.1 \pm 0.5$ & $5.6 \pm 0.8$ & $3.1 \pm 0.2$ & $3.2 \pm 0.2$ & $6.5 \pm 0.4$ & $6.4 \pm 0.3$ & $0.8 \pm 0.0$ & $0.7 \pm 0.1$ & $4.3 \pm 0.3$ & $4.4 \pm 0.3$ & $8.4 \pm 0.9$ & $7.7 \pm 1.1$ & $4.1 \pm 0.3$ & $3.3 \pm 1.5$ & $9.3 \pm 1.1$ & $7.5 \pm 3.8$ \\
\hline 5 & $5.7 \pm 0.6$ & $5.8 \pm 0.9$ & $3.2 \pm 0.1$ & $3.0 \pm 0.3$ & $6.4 \pm 0.4$ & $6.3 \pm 1.0$ & $0.8 \pm 0.1$ & $0.7 \pm 0.1$ & $4.5 \pm 0.2$ & $4.6 \pm 0.4$ & $7.6 \pm 1.0$ & $7.7 \pm 1.6$ & $3.7 \pm 0.3$ & $3.2 \pm 1.3$ & $8.4 \pm 0.9$ & $6.8 \pm 3.8$ \\
\hline 10 & $6.3 \pm 0.6$ & $6.6 \pm 0.6$ & $3.0 \pm 0.2$ & $2.8 \pm 0.4$ & $6.5 \pm 0.3$ & $6.3 \pm 1.0$ & $0.8 \pm 0.1$ & $0.7 \pm 0.1$ & $4.5 \pm 0.3$ & $4.5 \pm 0.2$ & $8.8 \pm 0.9$ & $9.2 \pm 0.2$ & $4.1 \pm 0.3$ & $2.9 \pm 1.0$ & $9.8 \pm 0.4$ & $5.5 \pm 2.5$ \\
\hline 15 & $6.0 \pm 0.6$ & $6.0 \pm 0.6$ & $3.2 \pm 0.3$ & $2.9 \pm 0.4$ & $6.4 \pm 0.6$ & $6.4 \pm 0.8$ & $0.8 \pm 0.1$ & $0.7 \pm 0.1$ & $4.5 \pm 0.2$ & $4.6 \pm 0.4$ & $8.4 \pm 0.6$ & $9.0 \pm 0.9$ & $4.0 \pm 0.3$ & $3.3 \pm 1.3$ & $9.1 \pm 0.8$ & $7.2 \pm 2.9$ \\
\hline 20 & $6.6 \pm 0.9$ & $5.8 \pm 0.8$ & $3.1 \pm 0.2$ & $2.9 \pm 0.3$ & $6.4 \pm 0.4$ & $6.5 \pm 0.8$ & $0.8 \pm 0.1$ & $0.7 \pm 0.1$ & $4.4 \pm 0.2$ & $4.5 \pm 0.2$ & $9.1 \pm 1.2$ & $8.2 \pm 0.9$ & $3.9 \pm 0.4$ & $3.1 \pm 1.2$ & $8.8 \pm 0.9$ & $6.3 \pm 2.7$ \\
\hline 25 & $6.0 \pm 0.2$ & $6.2 \pm 0.8$ & $3.1 \pm 0.2$ & $3.3 \pm 0.4$ & $6.4 \pm 0.5$ & $6.1 \pm 1.3$ & $0.8 \pm 0.0$ & $0.7 \pm 0.1$ & $4.5 \pm 0.2$ & $4.6 \pm 0.1$ & $7.8 \pm 0.4$ & $8.4 \pm 1.2$ & $3.5 \pm 0.6$ & $2.9 \pm 1.0$ & $8.3 \pm 1.0$ & $5.9 \pm 3.2$ \\
\hline 30 & $6.1 \pm 0.9$ & $5.7 \pm 0.5$ & $3.1 \pm 0.2$ & $2.9 \pm 0.6$ & $6.5 \pm 0.4$ & $6.2 \pm 1.0$ & $0.8 \pm 0.1$ & $0.6 \pm 0.2$ & $4.4 \pm 0.3$ & $4.3 \pm 0.2$ & $8.2 \pm 1.0$ & $8.1 \pm 0.5$ & $3.8 \pm 0.5$ & $3.0 \pm 1.3$ & $8.6 \pm 1.0$ & $6.5 \pm 3.1$ \\
\hline 35 & $6.5 \pm 0.6$ & $5.5 \pm 0.6$ & $3.1 \pm 0.1$ & $3.0 \pm 0.3$ & $6.2 \pm 0.4$ & $6.6 \pm 0.6$ & $0.8 \pm 0.0$ & $0.7 \pm 0.1$ & $4.4 \pm 0.2$ & $4.5 \pm 0.3$ & $8.8 \pm 0.6$ & $7.8 \pm 0.7$ & $3.5 \pm 0.3$ & $2.9 \pm 1.0$ & $8.0 \pm 1.0$ & $6.5 \pm 2.3$ \\
\hline 40 & $6.1 \pm 0.5$ & $5.9 \pm 0.5$ & $3.1 \pm 0.2$ & $3.0 \pm 0.4$ & $6.4 \pm 0.2$ & $6.4 \pm 0.6$ & $0.8 \pm 0.1$ & $0.7 \pm 0.1$ & $4.4 \pm 0.1$ & $4.6 \pm 0.3$ & $8.6 \pm 0.5$ & $8.3 \pm 0.7$ & $3.8 \pm 0.4$ & $2.8 \pm 1.2$ & $9.3 \pm 1.0$ & $6.1 \pm 3.0$ \\
\hline 45 & $6.2 \pm 0.4$ & $6.3 \pm 0.7$ & $3.1 \pm 0.1$ & $2.7 \pm 0.4$ & $6.7 \pm 0.5$ & $5.6 \pm 1.3$ & $0.8 \pm 0.1$ & $0.6 \pm 0.1$ & $4.4 \pm 0.3$ & $4.1 \pm 0.9$ & $8.9 \pm 0.6$ & $8.5 \pm 1.4$ & $3.8 \pm 0.4$ & $3.0 \pm 1.4$ & $9.3 \pm 1.7$ & $7.4 \pm 3.4$ \\
\hline
\end{tabular}




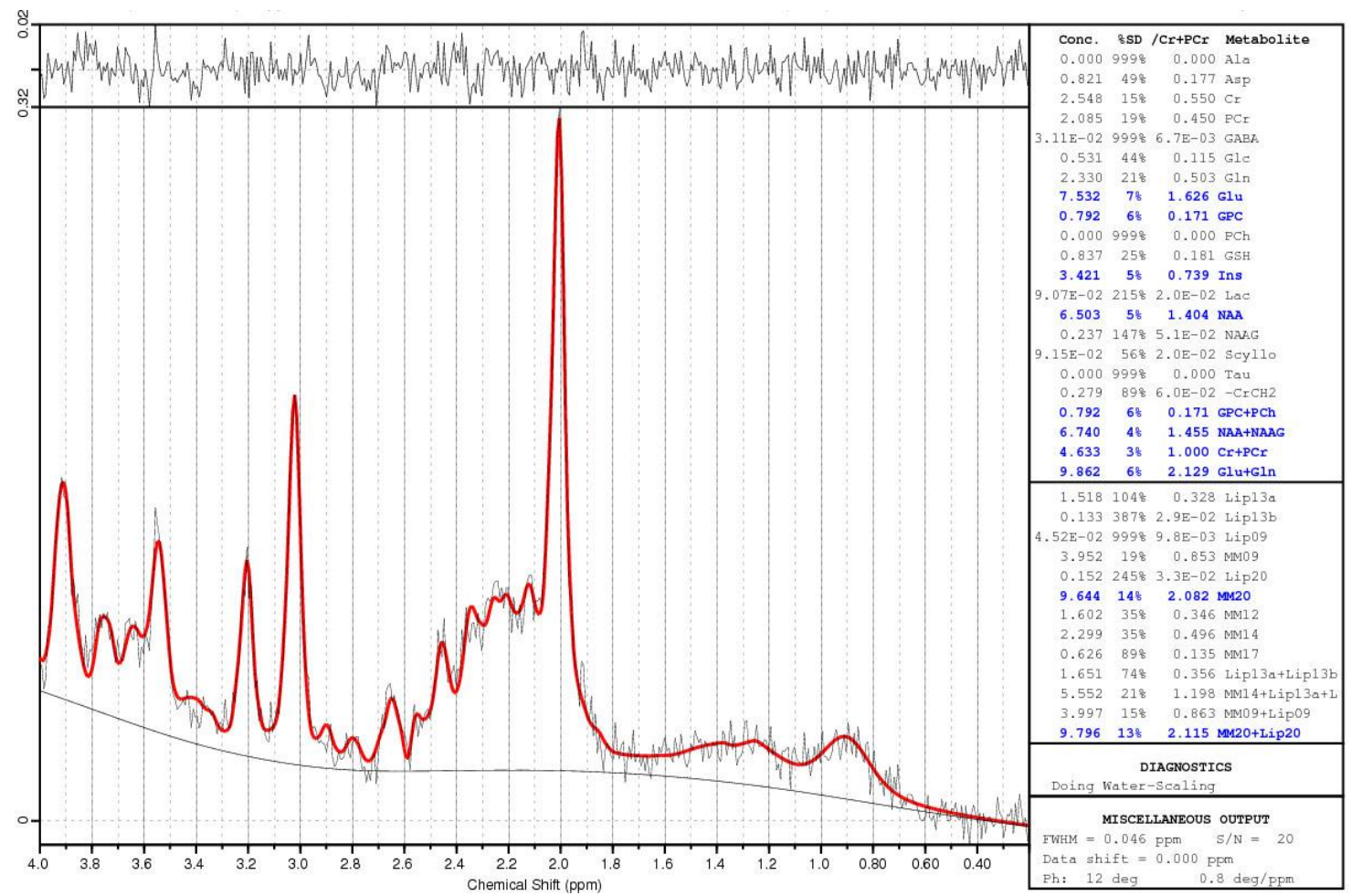

Fig. 1. Example of LCModel analysis of a proton magnetic resonance (1H-MR) spectrum acquired from bi-occiptalgray matter from a control participant. Analysis was undertaken using an appropriate simulated basis set

Although there was a significant time effect for GIx ( $P=0.04)$ (Figs. $2 \mathrm{c}$ and $2 \mathrm{~d}$ ), no group by time effect was found $(P>.05)$. There was larger variability as evidenced by the large standard deviation in concentrations for all metabolites for the intervention group) compared to the control group.

\section{DISCUSSION}

This is the first study to explore changes in brain metabolites using MRS following the consumption of a commercial ED and compared with a no drink control. This study demonstrated that the consumption of ED within Australian guidelines for maximum safe levels of caffeine had differing effects on the brain metabolites NAA and MM20. Over a 60 minute time frame absolute concentrations of NAA and MM20 decreased after ED consumption. The changes detected in this study demonstrate the sensitivity of the MRS method even when measured with 1.5T magnet. Consumption of two cans of ED were found to produce few changes with a moderate effect size for NAA (Cohensd). The study provides insight into an understudied, topical area of research whereby nutritional claims for energy drinks are often highly debated in the literature. The use of MRS in this study provides an objective measure to assess short term changes in brain chemistry following ED consumption. Additionally, MRS did not appear to pose a recruitment issue for this small exploratory study.

There are increasing concerns as to the potential effects of combining alcohol with ED's [43,44], as was reported by the majority of ED consumers in this study. It has been previously reported that stimulant ingredients such as caffeine and guarana in ED's dull the depressant effects of alcohol [23]. This includes giving the consumer a false sense of physical and mental competence, decreasing awareness of alcohol-related impairment [21-23,43], increasing amounts of alcohol consumed $[45,46]$ and increasing the likelihood of engaging in risk taking behaviours [47-51]. 


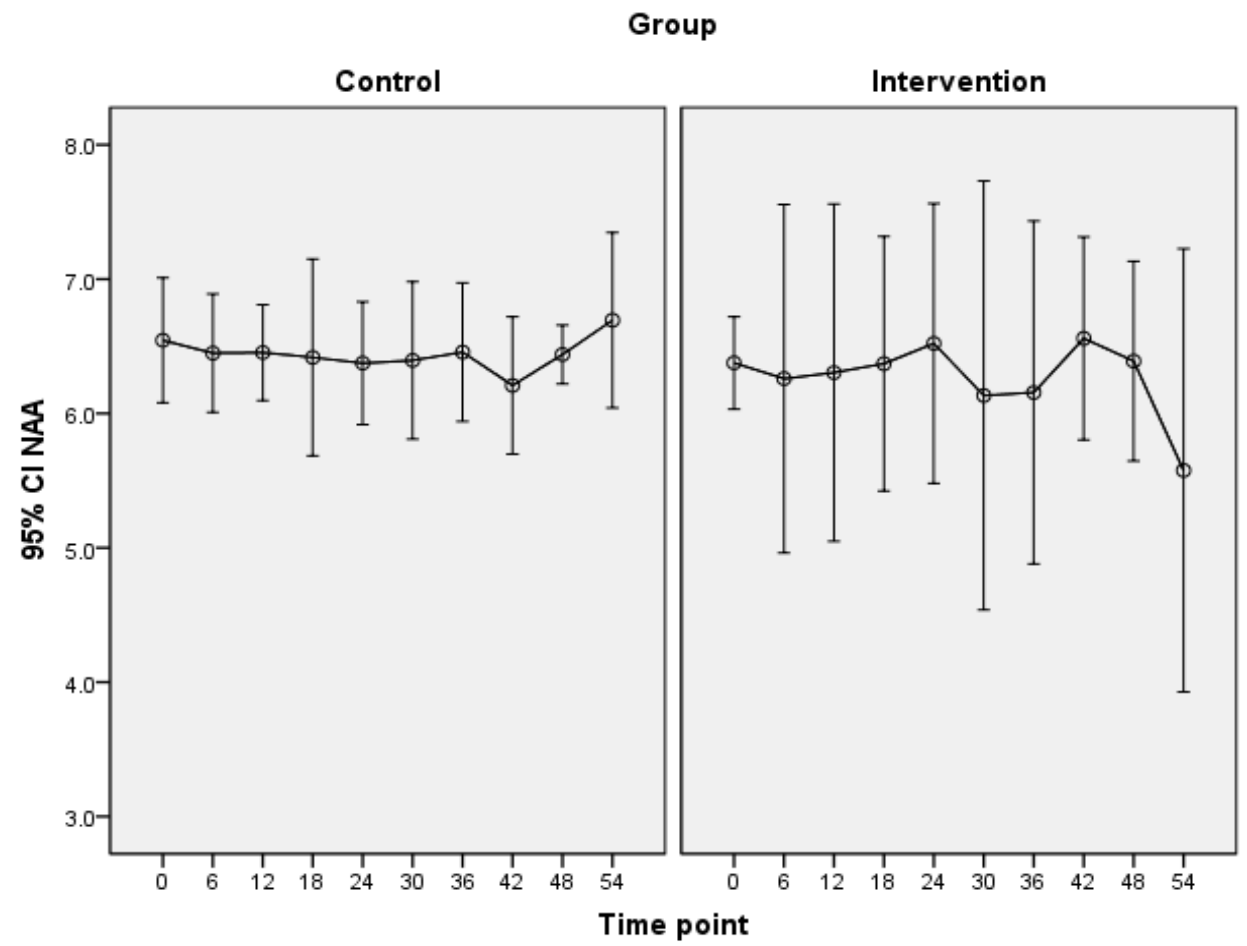

Fig. 2a. Mean concentrations of $\mathrm{N}$-acetylaspartate (NAA) by group over time. Values shown are $\mathrm{mmol} / \mathrm{kg}$. Error bars represent the $95 \%$ confidence interval

Group

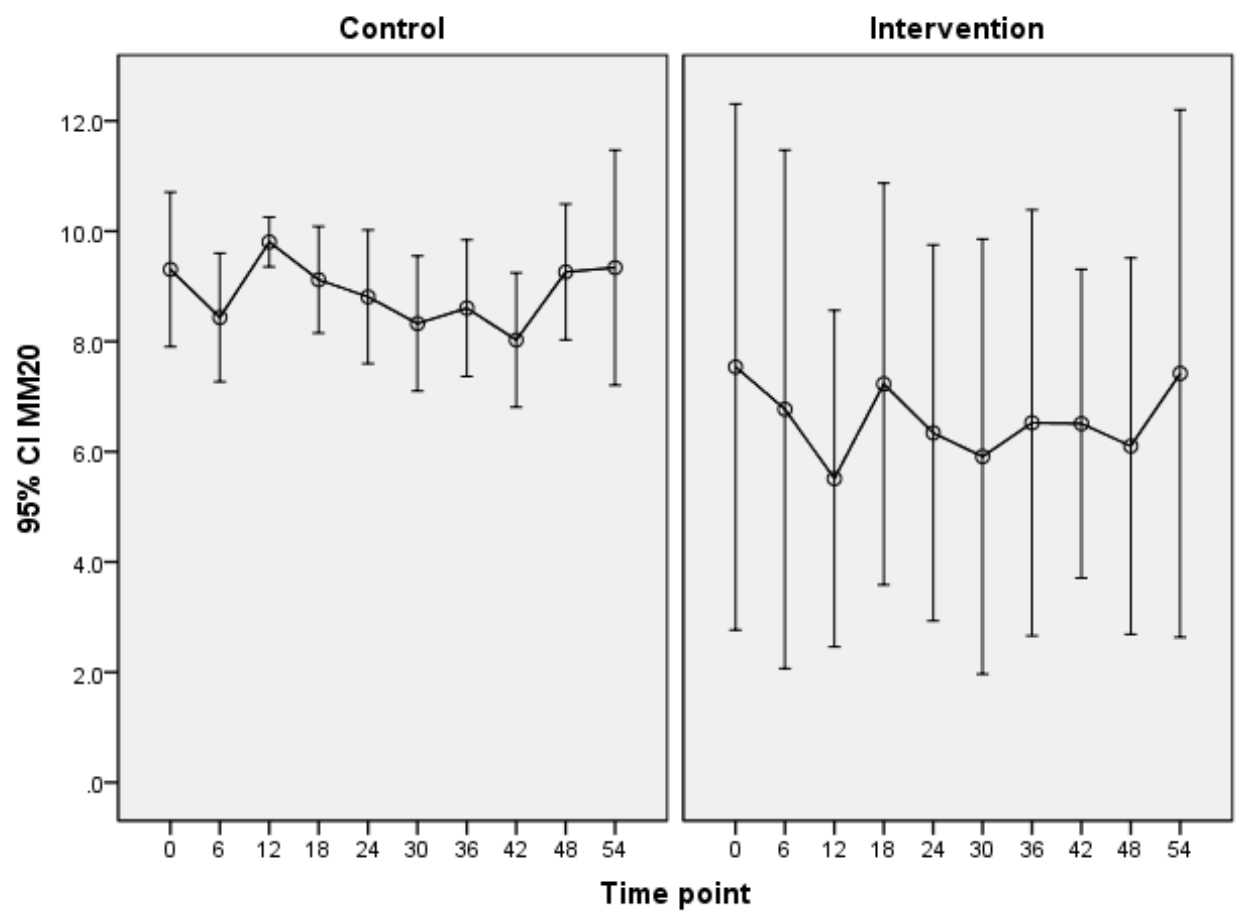

Fig. 2b. Mean concentrations of Macromolecules (MM20) by group over time. Values shown are $\mathrm{mmol} / \mathrm{kg}$. Error bars represent the $95 \%$ confidence interval 


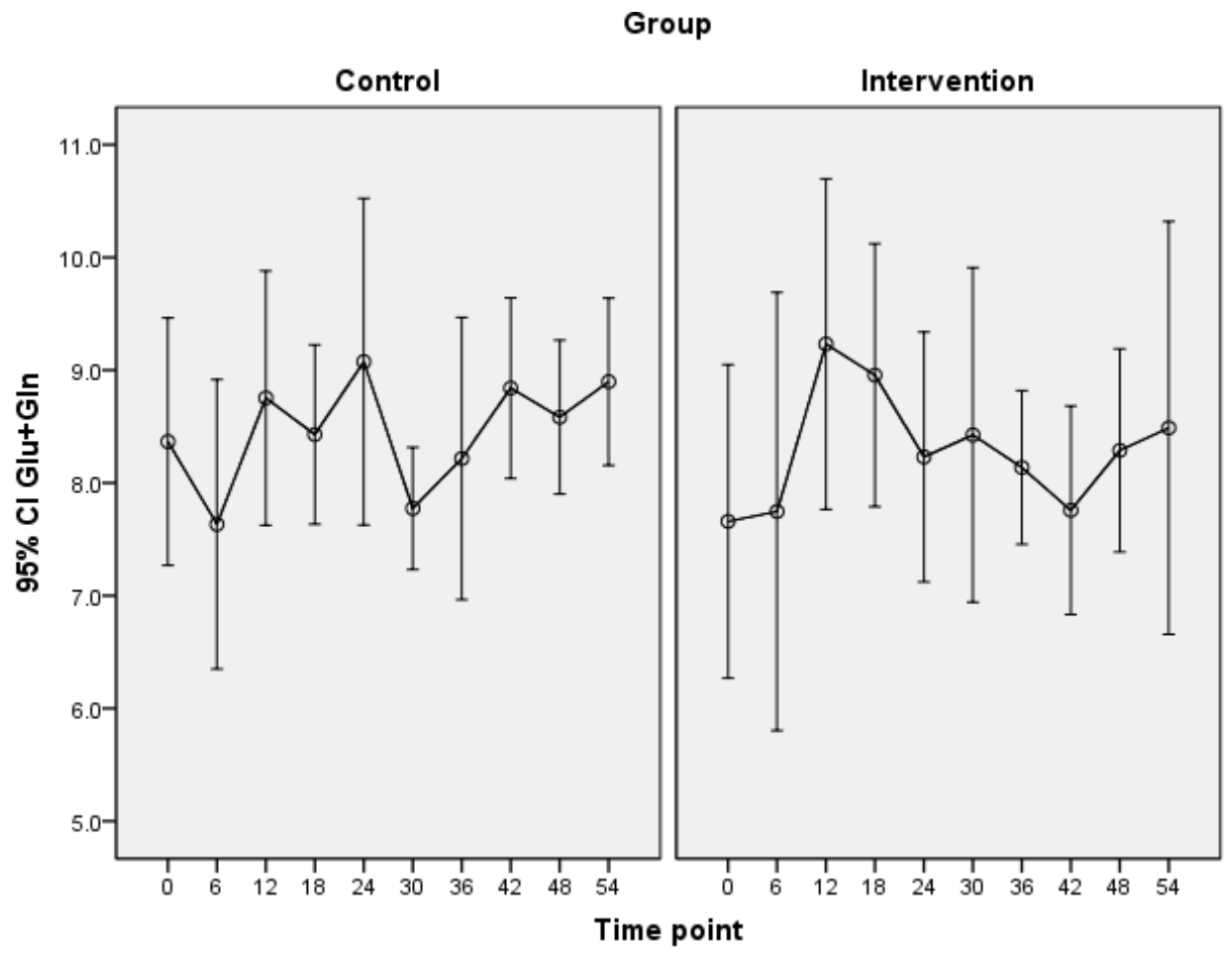

Fig. 2c. Mean concentrations of Glutamate+Glutamine (GIx) by group over time. Values shown are $\mathrm{mmol} / \mathrm{kg}$. Error bars represent the $95 \%$ confidence interval

Group

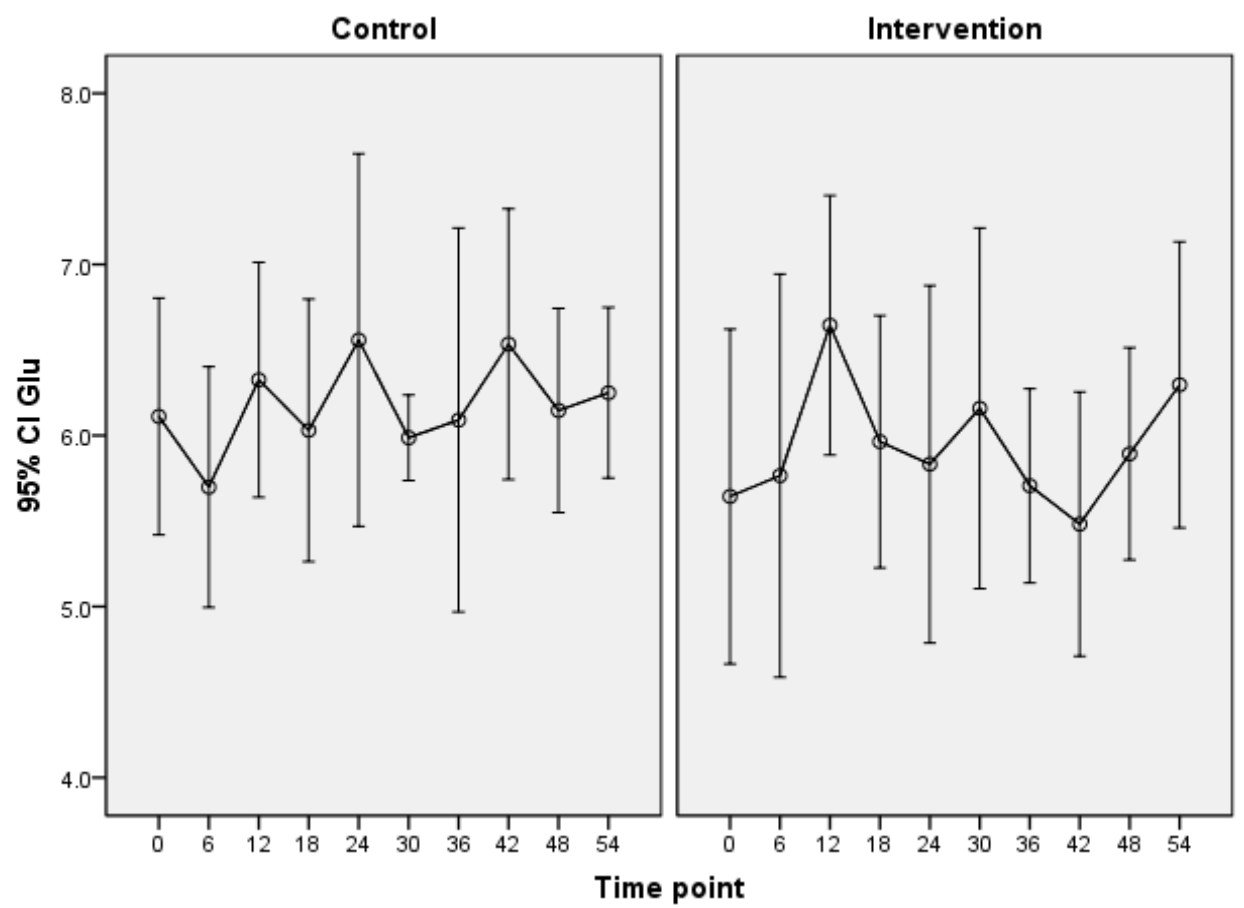

Fig. 2d. Mean concentrations of Glutamate (Glu) by group over time. Values shown are $\mathrm{mmol} / \mathrm{kg}$. Error bars represent the $95 \%$ confidence interval 
$\mathrm{N}$-acetylaspartate (NAA) is generally considered a putative neuronal marker with its almost exclusive localisation in neurons, making it in a reliable diagnostic marker. However, NAA is additionally believed to play a role in osmoregulation and can be released to the interstitial space upon osmotic challenge [37] Nacetylaspartate was found to decrease in this study after ED consumption. While statistically significant, the absolute change was still within reference values. It is also possible the changes in NAA observed here are due primarily to changes in $\mathrm{N}$-acetylaspartylglutamate (NAAG), which is difficult to separate from NAA at the magnetic field strength employed in this study. Glutamate is the dominant excitatory amino acid in the human brain and thus is thought to have a role linking excitatory neurotransmission to energy production and usage [37]. Glutamine is synthesized from glutamate by glutamine synthetase in glia and converted back to glutamate by glutaminase in neurons, completing the glutamate-glutamine cycle. Both glutamate and glutamine are engaged in a number of important metabolic pathways like energy provision, synthesis of important chemicals like $\mathrm{N}$-acetylaspartate (NAA), N-acetylaspartylglutamate (NAAG), $\mathrm{Y}$-aminobutyrate (GABA) and glutathione (GSH), and protein synthesis [52]. Glutamine is commonly assessed in combination with glutamate (glutamate + glutamine) and referred to as Glx. Myo-inositol is an isomer of inositol and has been associated with osmolarity control in the human brain [37]. Cholinecontaining compounds are essential for membrane lipid synthesis and act as precursors for the biosynthesis of the neurotransmitter acetylcholine [53].

Direct comparison with other studies is difficult due to the lack of studies in this area, differences in measurement methodologies such as differing strengths of magnets, gradient performance and field homogeneity and also the reporting of brain metabolites as different units such as ratios with total creatinine $(\mathrm{tCr})$. The decrease in MM20 in participants who consumed the ED was surprising. These resonances are believed to be attributable to proteins and MM present in brain tissue [54], and be primarily assigned to methyl and methylene resonances of protein amino acids such as leucine [55]. It therefore possible that the decrease observed in this study could be explained by the presence of Red Bull ingredients that co-resonate with these macromolecules and therefore added destructively to attenuate the MM20 signal.
This study articulates the MRS sequencing procedures that could be applied to assess the impact of other foods and beverages. With a plethora of functional foods and beverages appearing on supermarket shelves internationally, the application of new objective methodologies such as MRS to the field of nutrition could offer insights into their effects after short and long term consumption [5].

Taurine was not reliably detected in this study as evidenced by CRLB $>20 \%$ using LCModel and metabolite basis set of $35 \mathrm{~ms}$. This does not support previous findings where a large elevation in taurine $(n=7)$ was observed 25 minutes following consumption of $750 \mathrm{~mL}$ of Red Bull[29]. Changes of the magnitude reported by Roser et al (1998) could have a number of potential implications for the normal functioning of the brain and osmoregulation [56]. Roser et al noted that the cerebellum was more sensitive for detecting taurine elevation rather than the bioccipital gray matter measured in this study. This may have limited the ability to detect a difference in taurine levels to the magnitude of the previous study.

The main limitation of this study are the small sample size. However, the number of participants in the study are in line with other exploratory studies $[5,57]$. As a random convenience sample of young adult males was recruited, it is likely that this limited any age or gender differences which have been observed for some brain metabolites [5]. This reduces the generalizability of the study findings to other population groups and ethnicities. A limitation of the study was that the control group consumed no drink. This unbalanced intake of water may have led to differences in the concentration of brain metabolites. In future studies using MRS to identify differences in brain metabolites following intake of beverages should use a control beverage matched for fluid composition. Full demographic details of the participants and their educational ability were not collected as part of the study.

Although this study assessed usual consumption of ED, habitual caffeine consumption including commonly consumed products such as tea, coffee, cola and chocolate was not. Chronic caffeine consumption often leads to an increase in adenosine receptor density and metabolism $[58,59]$. Adenosine receptors function in the brain to regulate neurotransmitters including dopamine and glutamate $[60,61]$. Therefore it is possible that metabolite concentrations identified in this 
study could have been affected by individual differences in long term consumption patterns of other caffeinated beverages more broadly. Future studies using MRS to investigate the effects of food and beverages on the brain should assess habitual caffeine consumption of study subjects more comprehensively. Additionally, smoking status of subjects was not assessed in this pilot study. It is well documented that the CYP1A2 enzyme catalyses the metabolism of numerous compounds including caffeine [62,63]. CYP1A2 expression is affected by a number of dietary and environmental factors including smoking, which enhances the metabolism and clearance of caffeine $[64,65]$. Future MRS studies should assess smoking status when investigating caffeinated foods and beverages as this could influence the interpretation of the study findings.

The strengths of the current study are that it assessed ED consumption at baseline, included a control group and that it used a strong statistical approach to assess any changes that may have occurred in brain metabolites after ED consumption. Variables that influence metabolite concentration include echo time (TE), repetition time (TR), population variables (age, gender, brain location) so acquiring our own control data with the same acquisitionvariables in a similar population group is unique. Future studies should investigate the effects of ED's when combined with alcohol, utilize larger sample sizes, and a more culturally diverse population as well as using higher strength magnets that improve spectral resolution and thus metabolite identification and quantification [37]. Additionally, participants received a pre-scan nutrition protocol including fasting for two hours prior to scanning and avoidance of caffeine and alcohol products ten hours prior to scanning. Future studies may consider providing more extensive nutritional standardisation for the days leading into scanning to further control variables related to dietary intake and effects on brain metabolites.

The findings of the study including adaptation of the methodology may be used to generate meaningful power size calculations to detect possible changes and inform future research into the effects of other foods and beverages could have on brain metabolites. This is important given a recent review has highlighted the benefits of brain imaging procedures such as MRS in nutrition intervention studies [5].

\section{CONCLUSION}

This exploratory study found that the consumption of two cans of ED produced few changes in specific brain metabolites including taurine, NAA, Glx, and MM20 with moderate effect sizes. Changes detected in this study demonstrate the sensitivity of the MRS method to explore nutrition related variables with $1.5 \mathrm{~T}$ clinical MRI unit. The non-invasive quality of MRS make it a highly valuable technique and investigation of the effects of ED on brain metabolites is only one of many paradigms that could be investigated. Other protocols conducted over a longer duration or using higher dosages or in combination with alcohol may produce different results and is worthy of future study. Given the increasing consumption of ED, this study provides some insight into a topical area of research.

\section{CONSENT}

All authors declare that 'written informed consent was obtained from the patient (or other approved parties) for publication of this case report and accompanying images.

\section{ETHICAL APPROVAL}

All authors hereby declare that all experiments have been examined and approved by the appropriate ethics committee and have therefore been performed in accordance with the ethical standards laid down in the 1964 Declaration of Helsinki. The study was approved by the University of Newcastle Human Research Ethics Committee, Approval No. H- [20110034].

\section{ACKNOWLEDGEMENTS}

This research was funded by an ECR grant for T Burrows University of Newcastle. The authors thank Dr Saad Ramadan his technical assistance in the acquisition of data.

\section{COMPETING INTERESTS}

There are no competing interests to declare in this study.

\section{REFERENCES}

1. Duarte JMN, et al. The neurochemical profile quantified by in vivo $1 \mathrm{~h} \mathrm{nmr}$ spectroscopy. 2012;61(2):342-362. 
2. Mountford CE. et al. Neurospectroscopy: The past, present and future. Chemical Reviews. 2010;110(5):3060-3086.

3. Kreis $R$. et al. reproducibility of cerebral phenylalanine levels in patients with phenylketonuria determined by $1 \mathrm{~h}-\mathrm{mr}$ spectroscopy. Magnetic Resonance in Medicine. 2009;62(1):11-16.

4. Mangia $S$, et al. Neurochemical profile of patients with type 1 diabetes measured by $1 \mathrm{~h}-\mathrm{mrs}$ at $4 \mathrm{t}$. Journal of Cerebral Blood Flow \& Metabolism. 2013;33(5):754-759.

5. Sizonenko $S$, et al. brain imaging and human nutrition: Which measures to use in intervention studies. British journal of nutrition. 2013;110(s1).

6. Terpstra $M$, et al. noninvasive quantification of human brain antioxidant concentrations after an intravenous bolus of vitamin c. $\mathrm{Nmr}$ in biomedicine. 2011;24(5):521-528.

7. Smith FE, et al. Quantitative lithium magnetic resonance spectroscopy in the normal human brain on a $3 \mathrm{t}$ clinical scanner. Magn Reson Med. 2011;66(4):945-949.

8. Triebel S, et al. Rapid analysis of taurine in energy drinks using amino acid analyzer and fourier transform infrared (ftir) spectroscopy as basis for toxicological evaluation. Amino Acids. 2007;33(3):451457.

9. HeckmanMA KSherry, de mejia eg. Energy drinks: An assessment of their market size, consumer demographics, ingredient profile, functionality, and regulations in the united states. Compre Rev Food Sci F. 2010;9(3):303-317.

10. Baum $M$, Weiss $M$. The influence of a taurine containing drink on cardiac parameters before and after exercise measured by echocardiography. Amino Acids. 2001;20(1):75-82.

11. Geiß KR, et al. The effect of a taurinecontaining drink on performance in 10 endurance-athletes. Amino Acids. 1994;7(1):45-56.

12. Ivy $\mathrm{JL}$, et al. Improved cycling time-trial performance after ingestion of a caffeine energy drink. International Journal of Sport Nutrition and Exercise Metabolism. 2009;19(1):61-78.

13. Althea F. FDA warns caffeinated alcohol energy drinks are unsafe. National Journal; 2010.
14. Australia and new zealand food regulation ministerial council communique. [website]; 2011.

Availablefrom:http://www.foodstandards.go v.au/scienceandeducation/newsroom/medi areleases/mediareleases2011/australiaand newzeala5154.cfm

15. lyadurai SJP, Chung SS. New-onset seizures in adults: Possible association with consumption of popular energy drinks. Epilepsy Behav. 2007;10(3):504-508.

16. Röggla $\mathrm{G}$, et al. Seizures in a night club. The lancet. 2007;370(9583):220.

17. Machado-vieira $\mathrm{R}$,. Viale $\mathrm{Cl}$, Kapczinski F. Mania associated with an energy drink: The possible role of caffeine, Taurine and Inositol Can Journ Psychiat. 2001;46(5):454.

18. Nagajothi $\mathrm{N}$, et al. Energy drink-related supraventricular tachycardia. American Journal of Medicine. 2008;121(4):E3-4.

19. Terlizzi R, et al. Reversible postural tachycardia syndrome due to inadvertent overuse of red bull I. Clinical Autonomic Research. 2008;18(4):221-223.

20. Greene E, Oman K, Lefler M. Energy drink-induced acute kidney injury. Ann Pharmacother. 2014;48(10):1366-70.

21. Ferreira SE, et al. Effects of energy drink ingestion on alcohol intoxication. Alcohol Clin Exp Res. 2006;30(4):598-605.

22. Schoffl I, et al. Vodka Enegy: Too much for the adolescent nephron? Pediatrics. 2011;128(1):E227-e231.

23. Weldy DL. Risks of alcoholic energy drinks for youth. Journal of the American Board of Family Medicine. 2010;23(4):555-558.

24. Striley CW, khan SR. Review of the energy drink literature from 2013: Findings continue to support most risk from mixing with alcohol. Curr Opin Psychiatry. 2014;27(4):263-8.

25. Peacock $A$, et al. 'H'gh' risk? A systematic review of the acute outcomes of mixing alcohol with energy drinks. Addiction. 2014;109(10):1612-33.

26. Burrows $\mathrm{T}$, et al. What are the health implications associated with the consumption of energy drinks? A systematic Review. Nutrition Reviews. 2013;71(3):135-148.

27. Mclellant, Lieberman $\mathrm{H}$. Do energy drinks contain active components other than caffeine? Nutrition Reviews. 2012;70(12):730-744.

28. Shao A, Hathcock JN. Risk assessment for the amino acids taurine, I-glutamine and I- 
arginine. Regulatory Toxicology \& Pharmacology. 2008;50(3):376-99.

29. Roser $\mathbf{W}$, et al. Dangerous increase of taurine in the human brain after consumption of ' $\mathrm{n}$ 'energy dr'nk'? In proceedings to the 16th scientific annual meeting of the International Society for Magnetic Resonance in Medicine; 1998.

30. Maudsley A, et al. Mapping of brain metabolite distributions by volumetric proton $\mathrm{mr}$ spectroscopic imaging (mrsi). Magn Reson Med. 2009;61(3):548-559.

31. Smith PF, et al. Safety aspects of dietary caffeine - report from the expert working group. Australia New Zealand Food Authority; 2000.

32. Seng KY, et al. Population pharmacokinetics of caffeine in healthy male adults using mixed-effects models. Journal of Clinical Pharmacy and Therapeutics. 2009;34(1):103-114.

33. Pursey K, et al. Neural responses to visual food cues according to weight status: A systematic review of functional magnetic resonance imaging studies. Frontiers Nutrition; 2014.

34. Malinauskas BM, et al. A survey of energy drink consumption patterns among college students. Nutrition Journal. 2007;6:35.

35. Census of population and housing: Socioeconomic indexes for areas (Seifa). Australian bureau of statistics: Australia. P. Cat; 2011. No. 2033.0.55.001.

36. Kakeda ., et al. Influence of work shift on glutamic acid and gamma-aminobutyric acid (gaba): Evaluation with proton magnetic resonance spectroscopy at $3 t$. Psychiatry Research: Neuroimaging. 2011;192(1):55-59.

37. Duarte J, et al. The neurochemical profile quantified by in vivo $1 \mathrm{~h} \mathrm{nmr}$ spectroscopy. Neuroimage. 2012;61(2):342-62.

38. Provencher SW. automatic quantitation of localized in vivo $1 \mathrm{~h}$ spectra with Icmodel. Nmr in Biomedicine. 2001;14(4):260-264.

39. Cohen J. Statistical power analysis behavioural sciences 2nd ed, ed. N. Hillsdale. Lawrence Earlbaum and Associates; 1988.

40. Cole $\mathrm{T}$, et al. Establishing a standard definition for child overweight and obesity worldwide: International Survey. BMJ. 2000;320:1240.

41. Parks $M$, et al. Longituduinal brain metabolic characterization of chronic alcoholics with proton magentic resonance spectroscopy. Alcohol Clin Exp Res. 2002;26(9):1368-1380.

42. Govindaraju V, Young K, Maudsley A. Proton $\mathrm{nmr}$ chemical shifts and coupling constants for brain metabolites. Nmr Biomedicine. 2000;13:129-153.

43. Curry K, Stasio MJ. The effects of energy drinks alone and with alcohol on neuropsychological functioning. Human psychopharmacology, 2009;24(6):473-481.

44. Finnegan D. The health effects of stimulant drinks. Nutrition Bulletin. 2003;28(2):147155.

45. Arria $\mathrm{AM}$, et al. Energy drink consumption and increased risk for alcohol dependence. Alcoholism: Clinical and Experimental Research. 2011;35(2):365-375.

46. Velazquez CE, et al. Associations between energy drink consumption and alcohol use behaviors among college students. Drug and Alcohol Dependence. 2012;123(13):167-172.

47. Higgins JP, tuttle TD, Higgins CL. Energy beverages: Content and safety. Mayo Clinic Proceedings. 2010;85(11):10331041.

48. Pennay A, lubman DI, Miller P. Combining energy drinks and alcohol: $A$ recipe for trouble? Australian Family Physician. 2011;40(3):104-107.

49. Bigard AX. Risks of energy drinks in youths. Archives de Pediatrie. 2010;17(11):1625-1631.

50. Brache K, Stockwell T. Drinking patterns and risk behaviors associated with combined alcohol and energy drink consumption in college drinkers. Addictive Behaviors. 2011;36(12):1133-1140.

51. Snipes DJ, Benotsch EG. High-risk cocktails and high-risk sex: Examining the relation between alcohol mixed with energy drink consumption, sexual behavior, and drug use in college students. Addictive Behaviors. 2013;38(1):1418-1423.

52. Ramadan S, Lin A, Stanwell P. Glutamate and glutamine: A review of in vivo mrs in the human brain. $\mathrm{Nmr}$ in Biomedicine. 2013;26(12):1630-1646.

53. Mountford CE, et al. Neurospectroscopy: The past, present and future. Chem Rev. 2010;110:3060-3086.

54. Hoffman $L$, et al. Characterisation of the macromolecule localised h-mr spevtra of human brain. Magn Reson Med. 2001;46(5):855-863. 
55. Behar K, et al. Analysis of macromolecule resonances in $1 \mathrm{~h} \mathrm{nmr}$ spectra of human brain. Magn reson med. 1994;32(3):294302.

56. Pasantes-morales $H$, Schousboe A. Role of taurine in osmoregulation in brain cells: Mechanisms and functional implications. Amino Acids. 1997;12(3-4):281-292.

57. Babb SM, et al. Oral choline increases choline metabolites in human brain. Psychiatry Resear-h - Neuroimaging. 2004.;130(1):1-9.

58. Ribeiro JA, sebastião AM. Caffeine and adenosine. Journal of Alzhei'er's Disease. 2010;20(0):3-15.

59. Daly JW. Caffeine analogs: Biomedical impact. Cellular and molecular life sciences. 2007;64(16):2153-2169.

60. Ciruela $F$, et al. Presynaptic control of striatal glutamatergic neurotransmission by adenosine a1-a2a receptor heteromers. J Neurosci. 2006;26(7):2080-7.

61. Fredholm BB, et al. Actions of caffeine in the brain with special reference to factors that contribute to its widespread use. Pharmacol Rev. 1999;51(1):83-133.

62. Kalow W, Tang BK. Use of caffeine metabolite ratios to explore cyp1a2 and xanthine oxidase activities. Clin Pharmacol Ther. 1991;50(5 pt 1):508-19.

63. Butler MA, et al. Determination of cyp1a2 and nat2 phenotypes in human populations by analysis of caffeine urinary metabolites. Pharmacogenetics. 1992;2(3):116-27.

64. Kalow W, Tang BK. Caffeine as a metabolic probe: Exploration of the enzyme-inducing effect of cigarette smoking. Clin Pharmacol Ther. 1991;49(1):44-8.

65. Sachse $\mathrm{C}$, et al. Polymorphisms in the cytochrome p450 cyp1a2 gene (cyp1a2) in colorectal cancer patients and controls: Allele frequencies, linkage disequilibrium and influence on caffeine metabolism. British Journal of Clinical Pharmacology. 2003;55(1):68-76.

(c) 2015 Burrows et al.; This is an Open Access article distributed under the terms of the Creative Commons Attribution License (http://creativecommons.org/licenses/by/4.0), which permits unrestricted use, distribution, and reproduction in any medium, provided the original work is properly cited.

Peer-review history:

The peer review history for this paper can be accessed here: http://www.sciencedomain.org/review-history.php?iid= 853\&id=30\&aid=7395 